\title{
TWO-STEP APPROACH USING LUMPED PARAMETER AND FEM MODELS \\ FOR HAND AND ARM VIBRATION ANALYSIS
}

\author{
S. Pattnaik*, J. Kim \\ Department of Mechanical Engineering \\ University of Cincinnati \\ Cincinnati, Ohio 45221, U.S.A.
}

\section{Introduction}

Exposure to excessive vibration causes hand and arm vibration syndrome (HAVS) whose precise pathogenesis of HAVS remains unclear ${ }^{2}$. Population study and empirical tests provide useful but limited information; therefore numerical analysis is an attractive option if conducted properly. A lumped parameter based model appears to be more appropriate for analysis of low frequency vibration, while a finite element model is more appropriate for analysis of high frequency vibration. Therefore, a two-step approach that employs both models is proposed in this work. The lumped parameter model is used to estimate forces transmitted through joints tendons and supply basic data for the FEM model. The FEM model is used for further analysis of disorders in the vascular system and interphalangeal joints.

\section{Method \& Model}

Figure 1 shows a lumped parameter model of one of the fingers gripping a tool bar. Gripping forces have to be estimated from measurements or a separate kinematic analysis. The small spring-mass-damper system attached to each phalange represents the part of the finger in contact with the tool bar. $\mathrm{M}_{\mathrm{i}}$ and $\mathrm{I}_{\mathrm{i}}$ represent the mass and mass moment of inertia of the $i^{\text {th }}$ phalange. Circles specified as $\theta_{1}, \theta_{2}, \theta_{3}$ are interphalangeal joints modeled as small torsional spring and damped. Solid lines are flexor tendons and lumbrical muscles, and dash lines are inactive extensors. Due to redundancies built in the hand motion, exact analysis of individual

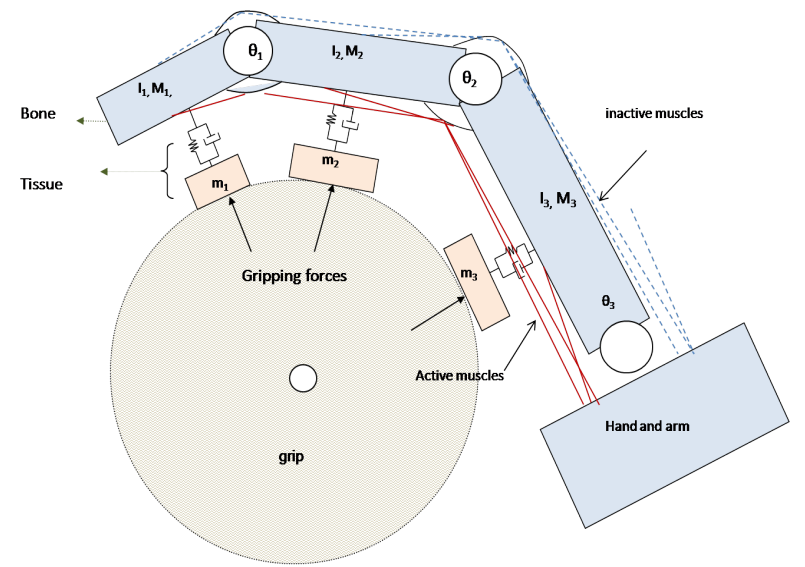

Fig. 1: Lumped parameter based power-grip tendons and muscles is impossible. As the static gripping is performed muscle recruiting begins with flexor profundus, followed by superficialis, finally with interossei and lumbricales as demand of force increases. Static analysis is conducted to identify active muscles, DC component of the joint force, and then followed by dynamic analysis. 


\section{Results / Discussion}

Figure 2 (left) shows the ADAMS model of the distal phalange, reduced from the model in Fig.1. The element shown as a long linear spring represents the flexor digitorum profundus, a long tendon attached to the upper arm. Effects of local muscles and synovial joints are lumped into a coil spring. Figure 2(right) shows undamped frequency responses calculated from the model. Solid line and dash-dot line represent the response at the tip of the bone with and without tendon, and dotted line is the response of the small muscle mass. Response shows three resonances between 10-100Hz.
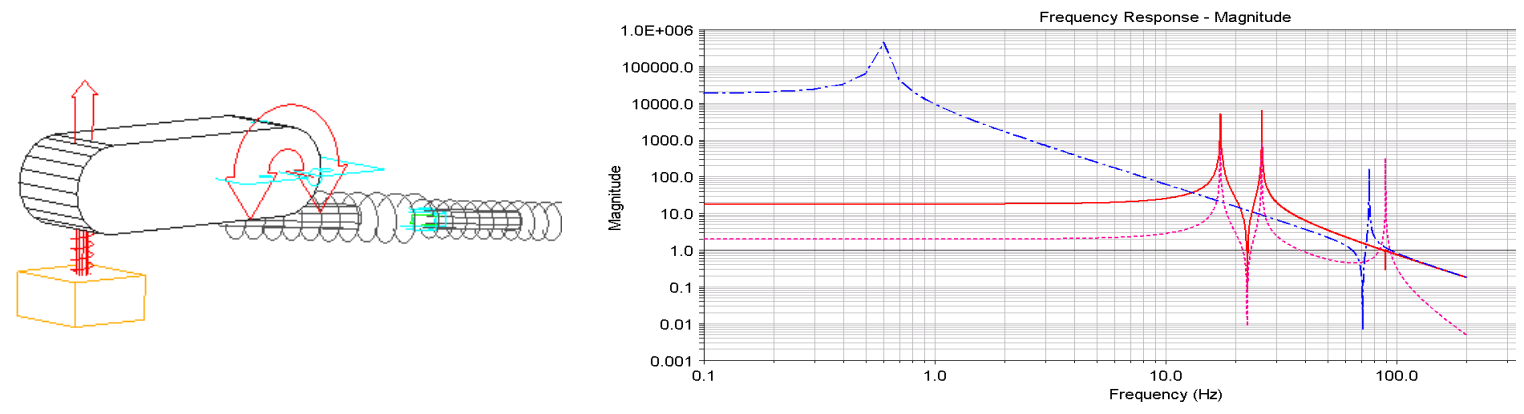

Fig. 2: (Left) ADAMS model (Right) Frequency responses

Figure 3 (left) shows a finite element model consists of distal and proximal segments of an index finger. Figure 3 (right) is the result of ABAQUS analysis that shows contours of the area deformed by $80 \%$ of the maximum deformation at various frequencies. It shows that the effect of vibration becomes more localized as the input frequency becomes higher.
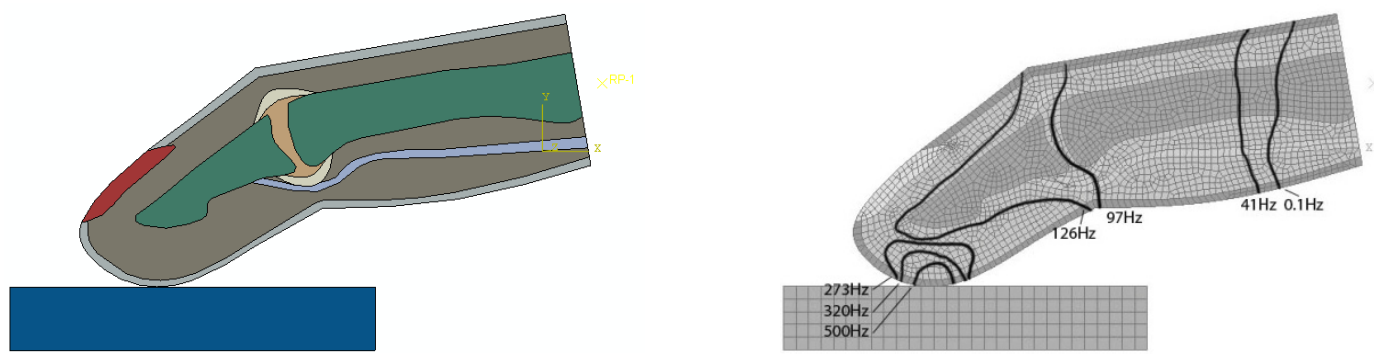

Fig. 3: (Left) Finite element model, (Right) Static deformation contour

\section{Future Work}

Estimating proper values of properties and developing a best procedure for the two-step analysis are necessary. Application of the results to understand effects of vibration on joints and vascular disorder is also planned.

\section{References}

1. Al Nazer and R, Rantalainen, T. (2008). Flexible multibody simulation approach in the analysis of tibian strain during walking. J. Biomechanics. Vol 41, Issue 5, pp 1036-104

2. Griffin, M. J. (1990). Handbook of human vibration. Academic Press

3. Wu, J. Z., Dong, R. G. and Welcome, D. E. (2006a). Analysis of the point mechanical impedance of fingerpad in vibration. Med Eng Phys 28 (8), 816-26.

4. Yamada, H. (1970). Strength of biological materials. Williams and Wilkins Co., Baltimore. 